\title{
Simulation Meets Experiment: Unraveling the Properties of Water in Metal-Organic
}

\section{Frameworks Through Vibrational Spectroscopy}

\author{
Kelly M. Hunter, ${ }^{*, \dagger}$ Jackson C. Wagner, ${ }^{\dagger}$ Mark Kalaj, ${ }^{\dagger}$ Wei Xiong, ${ }^{*, \dagger},+$ and \\ Francesco Paesani ${ }^{*},+, \uparrow, 9$ \\ $\dagger$ †epartment of Chemistry and Biochemistry, University of California San Diego, \\ La Jolla, California 92093, United States \\ $\ddagger$ Materials Science and Engineering, University of California San Diego, \\ La Jolla, California 92093, United States \\ ISan Diego Supercomputer Center, University of California San Diego, \\ La Jolla, California 92093, United States \\ E-mail: k6hunter@ucsd.edu; w2xiong@ucsd.edu; fpaesani@ucsd.edu
}

\begin{abstract}
In nanoporous materials, guest-host interactions affect the properties and function of both adsorbent and adsorbate molecules. Due to their structural and chemical diversity, metal-organic frameworks (MOFs), a common class of nanoporous materials, have been shown to be able to efficiently and, often, selectively adsorb various types of guest molecules. In this study, we characterize the structure and dynamics of water confined in ZIF-90. Through the integration of experimental and computational infrared (IR) spectroscopy, we probe the structure of heavy water $\left(\mathrm{D}_{2} \mathrm{O}\right)$ adsorbed in the pores, disentangling the fundamental framework-water and water-water interactions. The
\end{abstract}


experimental IR spectrum of $\mathrm{D}_{2} \mathrm{O}$ in ZIF-90 displays a blue-shifted OD-stretch band compared to liquid $\mathrm{D}_{2} \mathrm{O}$. The analysis of the IR spectra simulated at both classical and quantum levels indicates that the $\mathrm{D}_{2} \mathrm{O}$ molecules preferentially interact with the carbonyl groups of the framework and highlights the importance of including nuclear quantum effects and taking into account Fermi resonances for a correct interpretation of the OD-stretch band in terms of the underlying hydrogen-bonding motifs. Through a systematic comparison with the experimental spectra, we demonstrate that computational spectroscopy can be used to gain quantitative, molecular-level insights into framework-water interactions that determine the water adsorption capacity of MOFs as well as the spatial arrangements of the water molecules inside the MOF pores which, in turn, are key to the design of MOF-based materials for water harvesting.

\section{Introduction}

Hydrogen bonding (H-bonding) between water and host materials has proven to be important for the structure and function of the latter. ${ }^{1-3}$ Strong H-bonds can lead to the formation of extended networks that modulate fundamental processes, including hydration processes, ${ }^{4}$ chemical synthesis and reactions, ${ }^{5,6}$ heat dissipation,,${ }^{7,8}$ and macroscopic structural formations. ${ }^{7,9-11}$ Under confinement, H-bonded networks are disrupted due to physical constraints and host-water interactions. ${ }^{7,12}$ A variety of porous materials, such as carbon nanotubes, ${ }^{13-17}$ zeolites, ${ }^{18}$ silica pores and channels, ${ }^{12,19}$ and other materials ${ }^{20,21}$ have been used as model systems to determine the physical properties of water in confinement. These model systems highlight the fact that the properties of the confining environment, whether hydrophobic or hydrophilic, influence H-bonding with the host.

Metal-organic frameworks (MOFs) are an attractive class of porous materials. Comprised of inorganic subunits, also known as secondary building units (SBUs), and organic linkers, MOFs assemble in crystalline three-dimensional structures with large surface areas and high porosity. ${ }^{22}$ Because of their chemical diversity and tunability, MOFs have found a wide 
range of applications including, but not limited to, liquid and gas adsorption, ${ }^{23-26}$ chemical separation, ${ }^{27,28}$ catalysis, ${ }^{29}$ chemical degradation, ${ }^{30}$ and proton transport, ${ }^{31}$ as well as in electronic devices ${ }^{32}$ and magnets. ${ }^{33}$ It has recently been shown that some MOFs display remarkable water capture capabilities under ambient conditions. ${ }^{34-38}$ To further capitalize on the potential of MOFs as water harvesting materials, it is necessary to gain fundamental insights on the framework-water and water-water interactions in the confining pores, as these interactions determine both the onset and overall capacity of water adsorption.

An accurate description of the water H-bonding network in MOFs, which can allow for unambiguously disentangling framework-water and water-water interactions, is critical for understanding the physical mechanisms governing water adsorption in MOFs as a function of pore size and shape as well as the physicochemical properties of the framework. In this regard, an integrated approach that combines spectroscopic measurements and molecular simulations can provide such a level of detail. Linear infrared (IR) spectroscopy is sensitive to variations in the strength of the water H-bonding network, ${ }^{39}$ although all measurements are performed at the ensemble-averaged level, and the decoding of structure from the spectra is often challenging. On the other hand, molecular dynamics (MD) simulations provides a means to directly connect different spectroscopic features to specific H-bonding motifs. However, the calculated spectra, and, therefore, the reliability of the comparisons with the experimental measurements depend sensitively on the ability of the molecular models to correctly describe the underlying molecular interactions.

Diffuse reflection infrared Fourier transform spectroscopy (DRIFTS) can provide functionalgroup specific information regarding the interaction between water and the framework. A study carried out on MIL-53(Al), which exhibits the so-called "breathing effect", highlighted the complex mechanisms that modulate water adsorption in this MOF. ${ }^{40}$ It was determined that water interacts tightly with multiple sites of the framework at lower hydration levels, while at higher hydration levels the spectral signatures associated with the water $\mathrm{OH}$ stretching vibrations are localized to fewer sites of the framework. A study on the $\mathrm{Co}_{2} \mathrm{Cl}_{2} \mathrm{BTDD}$ 
MOF, a hydrophilic MOF that captures $82 \%$ water by weight below $30 \%$ relative humidity $(\mathrm{RH}),{ }^{36}$ used DRIFTS to elucidate the adsorption mechanism as a function of RH. ${ }^{41}$ Results from Ref. 41 provide a different picture from that derived from the analysis of the IR spectra of water in MIL-53(Al). Water was found to strongly bind to the open $\mathrm{Co}^{2+}$ sites of the framework at low RH and to subsequently form disconnected one-dimensional chains of Hbonded molecules bridging between the $\mathrm{Co}^{2+}$ sites. Upon further increase in $\mathrm{RH}$, these water chains were found to nucleate pore filling, with water molecules occupying the entire pore volume before the $\mathrm{RH}$ reaches $30 \%$. The different results from Refs. 40 and 41 exemplify not only the power of IR spectroscopy in providing functional group-specific information about the water adsorption process but also the variety of hydration mechanisms that can be observed in MOFs, depending on the pore sizes, shapes, and nature of the framework.

On the modeling side, one of the major challenges faced by MD simulations of MOFs is the development of force fields (FFs) that can accurately describe the underlying molecular interactions. Common FFs such as the general Amber force field (GAFF) ${ }^{42}$ and the universal force field (UFF) ${ }^{43}$ have been used to represent the organic linkers of MOFs. These FFs have the advantage of being applicable to a wide range of systems, although they suffer from a lack of transferability in describing the coordination and geometry of metal centers in MOFs. ${ }^{44,45}$ More specific FFs have been developed to address this deficiency, such as MOF-FF ${ }^{44}$ and QuickFF, ${ }^{46}$ extensions of UFF for MOFs (UFF4MOF), ${ }^{45,47}$ and a zeolitic imidazolate framework force field (ZIF-FF). ${ }^{48}$ These FFs have been extensively used to simulate adsorption isotherms as well as structural, thermodynamic, and dynamical properties of various molecules (e.g., methane and small hydrocarbons, carbon dioxide, and water) adsorbed in the MOF pores. Recently, more sophisticated polarizable models have also been used to model guest-framework interactions. ${ }^{49-54}$ Among different guest molecules, modeling water in MOFs presents particular challenges due to the complex nature of the water-water interactions, which is responsible for the anomalous behavior of water as a function of temperature and pressure. ${ }^{55-57}$ 
In this study, we integrate experimental and computational IR spectroscopy with MD simulations to investigate the properties of water in ZIF-90. Compared to most ZIFs that are highly hydrophobic (e.g., ZIF-8), ${ }^{58-60}$ ZIF-90 gives rise to a sharp step in the water adsorption isotherm between $30 \%$ and $40 \%$ RH (Fig. 1A) due to the presence of the imidazolate-2carboxyaldehyde linkers, which make the framework hydrophilic. ${ }^{61}$ While the overarching goal of this study is to gain general insights into the interplay between framework-water and water-water interactions, direct comparisons between experimental and simulated IR spectra also provide the unique opportunity to assess both merits and shortcomings of current models for MOF simulations and computational IR spectroscopy as well as to identify possible areas of improvement, which are critical for in silico screening of MOFs for water harvesting.

\section{Methods}

\section{Material Synthesis and Characterization}

ZIF-90 was synthesized by fully dissolving imidazole-2-carboxaldehyde (ICA) in N,N-dimethylformamide (DMF) with heat as described in Ref. 62. The ICA/DMF solution was cooled to room temperature, and pyridine was added. A solution of zinc nitrate hexahydrate in methanol was poured into the ICA/pyridine/DMF solution rapidly and mixed overnight. ZIF-90 crystals were collected through 4 cycles of centrifugation with neat DMF. The collected product was then washed with DMF and dried. To confirm the synthesis of ZIF-90, the product was analyzed using Powder X-Ray Diffraction (PXRD) and Scanning Electron Microscopy (SEM). PXRD data match well with theoretically simulated spectra, and obtained SEM images visually confirm the crystal structure of ZIF-90 (Figs. S1 and S2, respectively).

\section{Spectroscopic Measurements}

DRIFTS measurements were carried out on ZIF-90 crystal powder mixed with dry $\mathrm{KBr}$ at 5\% (w/w) using a Thermo Fischer Nicolet S10 FTIR spectrophotometer fitted with a PIKE 
Technologies DiffusIR accessory. The enclosed internal space of the DiffusIR accessory was purged with varying degrees of $\mathrm{D}_{2} \mathrm{O}$ humidified air using a humidity generator (Fig. S3). Briefly, dry air streams were mixed with $\mathrm{D}_{2} \mathrm{O}$ humidified air until the desired humidity was generated. The enclosed internal space of the accessory was then allowed to equilibrate for 10 minutes before each spectrum and background were recorded. A background spectrum of solid potassium bromide $(\mathrm{KBr})$ was taken for each $\mathrm{RH}$ value to correct for signal scatter using a Kubelka-Munk transform.

\section{Molecular Models and Simulations}

ZIF-90 was modeled using a flexible force field. All bonded parameters involving the zinc atom were taken from ZIF-FF, ${ }^{48}$ while all bonded parameters involving the atoms of the ligand were taken from the General Amber Force Field (GAFF). ${ }^{42}$ The MOF structure was optimized using periodic density functional theory (DFT) calculations carried out with the Vienna Ab initio Simulation Package (VASP) ${ }^{63-66}$ using the PBE exchange-correlation functional ${ }^{67}$ with Grimme's D3 dispersion correction $^{68}$ in combination with a projectoraugmented wave (PAW) treatment ${ }^{69,70}$ with a $700 \mathrm{eV}$ kinetic energy cutoff. A $2 \times 2 \times 2 k$-point grid was used, and forces were converged to a tolerance of $0.03 \mathrm{eV} / \AA$. Atomic point charges were calculated for the optimized ZIF-90 structure using the density derived electrostatic and chemical (DDEC) charges. $^{71}$ Lennard-Jones (LJ) van der Waals (VDW) parameters were taken from ZIF-FF with an initial guess used for the oxygen atom not present in ZIFFF. Atom types as well as all bonded and non-bonded parameters for the MOF force field are given in Fig. S4 and Tables S1-S4.

Water was modeled using the MB-pol potential energy function (PEF). ${ }^{72-74} \mathrm{MB}-$ pol has been shown to correctly predict the properties of water from the gas to the condensed phase ${ }^{75,76}$ which makes it particularly well-suited for modeling the properties of water adsorbed in MOFs as a function of RH. Furthermore, MB-pol enables accurate simulations of vibrational spectra of water clusters,${ }^{72,77,78}$ liquid water, ${ }^{79-82}$ the air/water interface, ${ }^{83,84}$ 
and ice, ${ }^{85-87}$ and is thus an ideal model to monitor the evolution of the DRIFTS spectra of water adsorbed in MOFs as a function of RH. ${ }^{41}$

As in Ref. 41, the framework-water non-bonded interactions were represented in terms of permanent electrostatics and Lennard-Jones (LJ) interactions. Since the extension of our many-body models to generic molecules compatible with MB-pol is under development, ${ }^{88,89}$ in this study the LJ parameters between the MOF atoms and water were obtained by applying the Lorentz-Berthelot mixing rules using the LJ parameters of the TIP4P/2005 water model, ${ }^{90}$ which is the closest point-charge model to MB-pol. ${ }^{91}$

All MD simulations were carried out in periodic boundary conditions for systems consisting of $2 \times 2 \times 2$ primitive cells of ZIF-90 and various water loadings, ranging from $25 \%$ to $70 \% \mathrm{RH}$, using in-house software based on the DL_POLY_2 simulation package. ${ }^{92}$ Constant pressure and constant temperature (NPT) simulations at 1.0 atm and $300 \mathrm{~K}$ were performed for 500 ps at each water loading. Since the average box dimensions do not deviate significantly from the experimental values (see Table S5 in the Supporting Information), the latter were used in all simulations to guarantee direct comparisons with the experimental measurements. Each system was equilibrated in the canonical (constant number of atoms, volume, and temperature, NVT) ensemble at $300 \mathrm{~K}$ for 1 ns. All dynamical properties, including the IR spectra, were calculated in the microcanonical (constant number of atoms, volume, and energy, NVE) ensemble at $300 \mathrm{~K}$ by averaging over 20, 50 ps long independent trajectories. The equations of motion were propagated according to the velocity-Verlet algorithm with a time step of $0.2 \mathrm{fs}$, and the temperature was maintained at $300 \mathrm{~K}$ by a Nosé-Hoover chain of four thermostats. Short-range interactions were truncated at an atom-atom distance of $15.0 \AA$, and long-range electrostatics were calculated using the Ewald sum. ${ }^{93}$

To assess the importance of nuclear quantum effects in determining the structural and dynamical properties of water adsorbed in ZIF-90, centroid molecular dynamics (CMD) simulations $^{94-98}$ were also performed. In the CMD simulations, each atom was represented by a Feynman's ring polymer discretized with 32 beads, and the centroid variables were propa- 
gated using the partially adiabatic separation scheme of Refs. 97 and 99, with an adiabaticity parameter of $\gamma=0.25$ and a time step of 0.05 fs. Given the associated computational cost, all CMD simulations were only performed for systems consisting of $1 \times 1 \times 1$ primitive cells of ZIF-90 at $40 \%$ RH.

The theoretical IR spectra were calculated within the time-dependent formalism according to

$$
I_{I R}=\left[\frac{2 \omega}{3 V \hbar c \epsilon_{0}}\right] \tanh (\beta \hbar \omega) \int_{-\infty}^{\infty} e^{i \omega t}\langle\mu(0) \mu(t)\rangle d t
$$

where $V$ is the system volume, $c$ is the speed of light in vacuum, $\epsilon_{0}$ is the permittivity of free space, and $\beta=\left(k_{B} T\right)^{-1}$, with $k_{B}$ being Boltzmann's constant. In Eq. $1,\langle\mu(0) \mu(t)\rangle$ is the ensemble-averaged dipole-dipole time correlation function that was calculated by averaging over 20 NVE trajectories at each water loading, with $\mu$ being represented by the MB- $\mu$ many-body dipole moment function. ${ }^{79}$

Due to the neglect of nuclear quantum effects, classical MD simulations predict vibrational spectra that are systematically blue-shifted relative to the corresponding experimental spectra. ${ }^{79,83,100,101}$ Therefore, in all the analyses presented in the following sections, the theoretical spectra calculated from classical MD simulations are red-shifted by $137 \mathrm{~cm}^{-1}$ in the stretching region, as shown in Fig. S5 in the Supporting Information, to facilitate comparisons with the experimental DRIFTS spectra. Furthermore, MB-pol has been shown to slightly underestimate the strength of H-bonds in liquid water, which results in a blue-shift of $57 \mathrm{~cm}^{-1}$ in the $\mathrm{OH}$ stretching region of the quantum CMD spectra. ${ }^{79}$ In the following analyses, the quantum CMD spectra are thus red-shifted by $57 \mathrm{~cm}^{-1}$ (Fig. S5) to facilitate comparisons with the experimental DRIFTS spectra.

Finally, Fermi resonances between the overtones of the bending vibrations and stretching vibrations of the water molecules in the liquid phase have been shown to be important for a quantitative representation of the OH-stretch lineshape in both water clusters ${ }^{77,102}$ and 
liquid water. ${ }^{82}$ To calculate the Fermi resonance contributions to the OD-stretch lineshape, local mode/local monomer (LM) calculations ${ }^{103-105}$ were performed on clusters comprised of a central $\mathrm{D}_{2} \mathrm{O}$ molecule surrounded by $16 \mathrm{H}_{2} \mathrm{O}$ molecules, which were used to model the intramolecular (bending and stretching) modes of $\mathrm{D}_{2} \mathrm{O}$ in the liquid phase. These clusters were the same clusters used in Ref. 82, which were extracted from CMD simulations of a single $\mathrm{H}_{2} \mathrm{O}$ in a $\mathrm{D}_{2} \mathrm{O}$ solution. The two hydrogen atoms on the $\mathrm{H}_{2} \mathrm{O}$ molecule were optimized, while every other atom was held fixed, using the ORCA package ${ }^{106}$ interfaced with our inhouse MB-pol software. After this optimization, the central $\mathrm{H}_{2} \mathrm{O}$ molecule was converted to $\mathrm{D}_{2} \mathrm{O}$ while the 16 surrounding $\mathrm{D}_{2} \mathrm{O}$ molecules in the cluster were converted to $\mathrm{H}_{2} \mathrm{O}$ for the LM calculations.

\section{Results and Discussion}

ZIF-90 exhibits a type $\mathrm{V}$ adsorption isotherm (Fig. 1A), ${ }^{60}$ adsorbing water in one porefilling step that begins at $\sim 30 \% \mathrm{RH}$ and ends at $\sim 40 \% \mathrm{RH}$. To facilitate the analysis of the IR spectra, all measurements and simulations were carried out with $\mathrm{D}_{2} \mathrm{O}$ since the OD stretching vibrations do not overlap with any of the framework vibrations. The experimental IR spectra of $\mathrm{D}_{2} \mathrm{O}$ in ZIF-90 in the OD stretching region, obtained after subtracting the
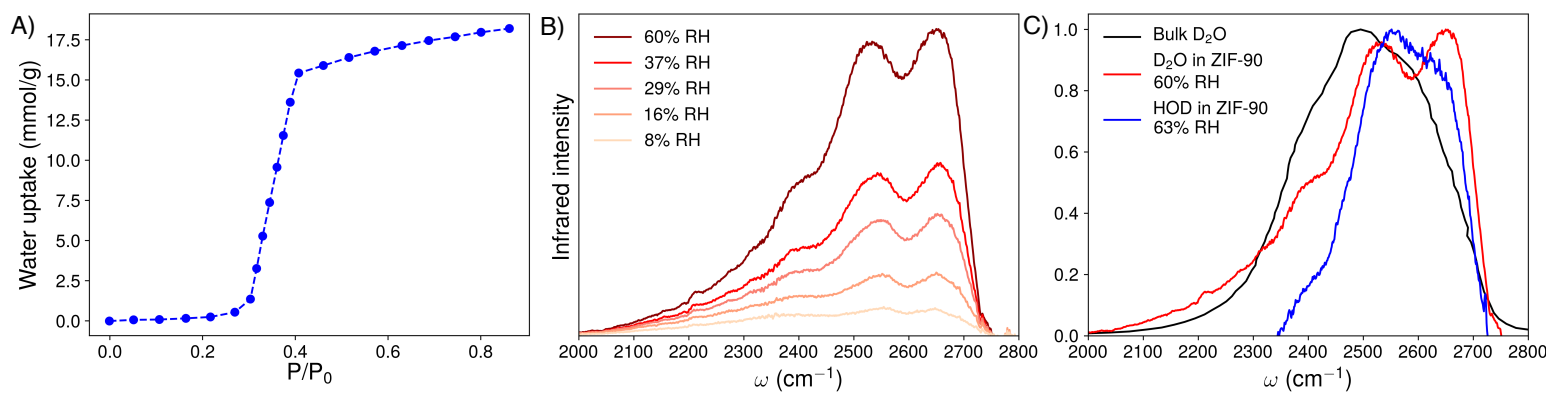

Figure 1: A) Water adsorption isotherm for ZIF-90 from Ref. 60. B) Experimental IR spectra of $\mathrm{D}_{2} \mathrm{O}$ in ZIF-90 at $8 \% \mathrm{RH}, 16 \% \mathrm{RH}, 29 \% \mathrm{RH}, 37 \% \mathrm{RH}$, and $60 \% \mathrm{RH}$. C) IR spectra of experimental bulk $\mathrm{D}_{2} \mathrm{O}$ (black) from Ref. $107, \mathrm{D}_{2} \mathrm{O}$ in ZIF-90 at $60 \% \mathrm{RH}$ (red), and HOD in ZIF-90 at 63\% RH (blue). The spectra are normalized to a value of one. 
spectrum measured at $0 \% \mathrm{RH}$ from the spectra measured at different $\mathrm{RH}$ values, are shown in Fig. 1B. As the humidity increases to $\sim 10 \%$, two peaks centered at $\sim 2550$ and $\sim 2650$ $\mathrm{cm}^{-1}$ begin to develop, with their intensity monotonically increasing as the RH increases. Importantly, Fig. 1B also shows that the spectral weight shifts from the lower to the higher frequency portion of the lineshape as the RH increases. The comparison with the IR spectrum of bulk $\mathrm{D}_{2} \mathrm{O}$ (Fig. 1C) indicates that ZIF-90 induces a blue shift of $\sim 40 \mathrm{~cm}^{-1}$ to the OD lineshape of the adsorbed $\mathrm{D}_{2} \mathrm{O}$ molecules. The OD lineshape in ZIF-90 displays appreciably higher intensity on the blue side of the maximum at $2650 \mathrm{~cm}^{-1}$. Importantly, DRIFTS measurements carried out for HOD in ZIF-90 (Fig. 1C) provide a significantly narrower OD-stretch lineshape that lacks the two distinct peaks that characterize the analogous ODstretch of $\mathrm{D}_{2} \mathrm{O}$ in ZIF-90. Furthermore, the absence of intramolecular coupling in the HOD molecules results in a decrease of the IR intensity on the red side of the spectrum around $\sim 2400 \mathrm{~cm}^{-1}$. The comparison of the IR spectra of $\mathrm{D}_{2} \mathrm{O}$ and HOD in ZIF-90 thus allows us to unambiguously assign the shoulder at $\sim 2400 \mathrm{~cm}^{-1}$ in the $\mathrm{D}_{2} \mathrm{O}$ spectrum to the Fermi resonances and not to particular ice-like structures of $\mathrm{D}_{2} \mathrm{O}$ in the ZIF-90 pores.

To gain insights into the adsorption process, IR spectra were calculated from both MD and CMD simulations carried out at different $\mathrm{D}_{2} \mathrm{O}$ loadings. Fig. 2 shows a comparison between the experimental, MD, and CMD OD-stretch lineshapes at $300 \mathrm{~K}$. As discussed in Ref. 82, due to numerical limitations associated with using Cartesian coordinates to propagate the dynamical trajectories, the lineshape calculated using quantum CMD simulations is unable to quantitatively describe Fermi resonances. ${ }^{108,109}$ On the other hand, the Fermi resonances do not appear in the lineshape calculated using classical MD simulations because, in this case, the overtones of the bending vibrations do not overlap with the OD stretching vibrations. Fig. 2A shows the experimental and simulated CMD IR spectrum of bulk $\mathrm{D}_{2} \mathrm{O}$. The CMD spectrum is able to reproduce the experimental $\mathrm{D}_{2} \mathrm{O}$ spectrum fairly well, only lacking intensity around $\sim 2400 \mathrm{~cm}^{-1}$, which is due to missing intensity from the Fermi resonances. ${ }^{82}$ This inability to reproduce Fermi resonances leads to two distinct peaks in the 

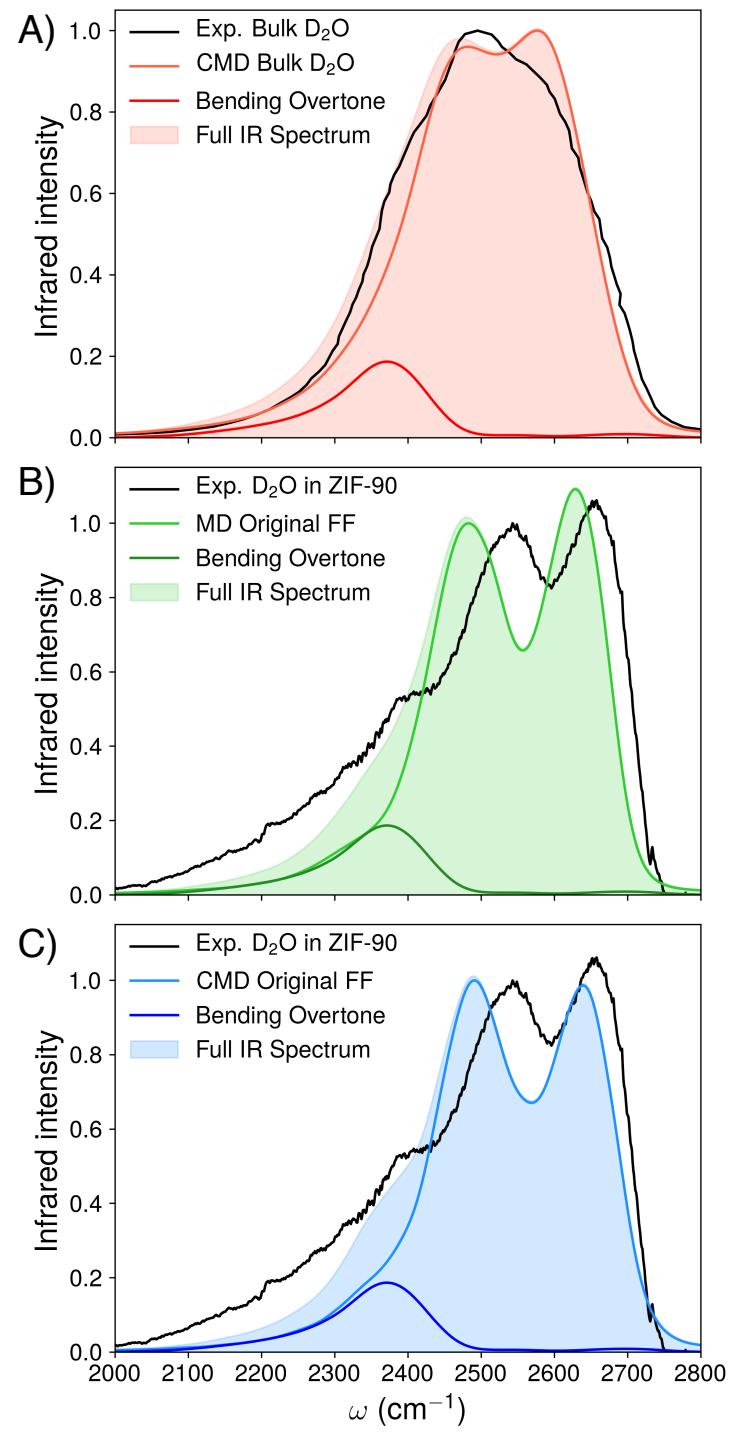

Figure 2: A) Experimental bulk $\mathrm{D}_{2} \mathrm{O}$ IR spectrum (black) from Ref. 107, CMD bulk $\mathrm{D}_{2} \mathrm{O}$ (pink) spectrum, Fermi resonance contribution (red), and CMD bulk $\mathrm{D}_{2} \mathrm{O}$ spectrum with added Fermi resonance contribution (shaded pink). B) Experimental IR spectrum of water in ZIF-90 at 40\% RH (black), MD 40\% RH (green) spectrum, Fermi resonance contribution (dark green), and MD 40\% RH spectrum with added Fermi resonance contribution (shaded green). C) Experimental IR spectrum of water in ZIF-90 at $40 \% \mathrm{RH}$ (black), CMD $40 \%$ RH (light blue) spectrum, Fermi resonance contribution (blue), and CMD $40 \%$ RH spectrum with added Fermi resonance contribution (shaded light blue). All MD spectra are red-shifted by $137 \mathrm{~cm}^{-1}$, CMD spectra are red-shifted by $57 \mathrm{~cm}^{-1}$, DOD bending overtones are blueshifted by $24 \mathrm{~cm}^{-1}$, and all spectra are normalized to a value of one at the lower frequency peak. 
CMD spectrum of bulk $\mathrm{D}_{2} \mathrm{O}$ instead of the single, broad peak with two shoulders observed in the experimental spectrum (Fig. 2A). After adding the contributions from the Fermi resonances (red trace), the resulting CMD OD-stretch lineshape of bulk $\mathrm{D}_{2} \mathrm{O}$ is in remarkably good agreement with the corresponding experimental lineshape.

Figs. 2B and 2C show comparisons between the experimental and theoretical lineshapes for $\mathrm{D}_{2} \mathrm{O}$ in ZIF-90 at $40 \% \mathrm{RH}$ calculated from both (classical) $\mathrm{MD}$ and (quantum) CMD simulations, respectively. While the simulated lineshapes correctly reproduce both the blue shift of the OD-stretch linesahpe measured experimentally (Fig. S7) and the presence of two distinct peaks, both MD and CMD lineshapes overestimate the separation between the two peaks compared to experiment.

The origin of the blue shift can be explained by investigating the structure of bulk $\mathrm{D}_{2} \mathrm{O}$ and $\mathrm{D}_{2} \mathrm{O}$ in ZIF-90. As shown in Table $1, \mathrm{D}_{2} \mathrm{O}$ molecules adsorbed in MOF pores form longer, and, consequently, weaker D-bonds compared to bulk. These longer D-bonds are associated with OD stretching vibrations that absorb at relatively higher frequencies, causing the blue shift of the OD-stretch lineshape found for $\mathrm{D}_{2} \mathrm{O}$ confined in the ZIF-90 pores.

Since it has been shown that MB-pol correctly describes the properties ${ }^{76}$ and IR spectrum of bulk $\mathrm{D}_{2} \mathrm{O}$ (Fig. 2A), the differences between the experimental and simulated IR spectra of $\mathrm{D}_{2} \mathrm{O}$ in ZIF-90 are likely due to an inaccurate representation of the interactions between the $\mathrm{D}_{2} \mathrm{O}$ molecules and the ZIF-90 framework. The present simulations (Fig. S9) as well as other studies of water in ZIF-90 ${ }^{59}$ indicate that the $\mathrm{D}_{2} \mathrm{O}$ molecules adsorbed in the ZIF-90 pores can D-bond to the carbonyl groups of the framework. Furthermore, Figs. 2B and 2C show that, compared to experimental results, the low-frequency peak in both MD and CMD ODstretch lineshapes is red-shifted and misses intensity around $\sim 2550 \mathrm{~cm}^{-1}$. This frequency region corresponds to $\mathrm{OD}$ stretching vibrations of $\mathrm{D}_{2} \mathrm{O}$ molecules that are D-bonded to the carbonyl groups of the framework. ${ }^{110}$

In order to more accurately describe the strength of D-bonding between the $\mathrm{D}_{2} \mathrm{O}$ molecules and the framework, we modified the LJ potential between the $\mathrm{D}_{2} \mathrm{O}$ oxygen atom $(\mathrm{OW})$ and 
Table 1: Average OD bond, water-water (OW-OW) D-bond lengths, and water-MOF (OWo) D-bond lengths calculated from MD and CMD simulations for bulk $\mathrm{D}_{2} \mathrm{O}$ as well as ZIF-90 at $40 \% \mathrm{RH}$ using both the original and modified force fields.

\begin{tabular}{lcccc}
\hline & Simulation & $\begin{array}{c}\text { OD bond } \\
(\AA)\end{array}$ & $\begin{array}{c}\text { OW-OW } \\
\text { D-bond }(\AA)\end{array}$ & $\begin{array}{c}\text { OW-o } \\
\text { D-bond }(\AA)\end{array}$ \\
\hline $\begin{array}{l}\text { Bulk D } 2 \mathrm{O} \\
\text { CMD }\end{array}$ & 0.976 & 1.916 & \\
\hline $\begin{array}{l}\text { ZIF-90 40\% RH } \\
\text { (original FF) }\end{array}$ & CMD & 0.973 & 1.937 & 2.146 \\
$\begin{array}{l}\text { ZIF-90 40\% RH } \\
\text { (original FF) }\end{array}$ & MD & 0.969 & 1.943 & 2.147 \\
\hline $\begin{array}{l}\text { ZIF-90 40\% RH } \\
(\text { modified FF) }\end{array}$ & CMD & 0.975 & 1.945 & 1.713 \\
$\begin{array}{l}\text { ZIF-90 40\% RH } \\
(\text { modified FF) }\end{array}$ & MD & 0.970 & 1.950 & 1.715 \\
\hline
\end{tabular}

the oxygen atom (o) of the carbonyl functional groups of the framework by decreasing $\sigma_{O W-o}$ by $10 \%$ and $\epsilon_{O W-o}$ by $20 \%$, which leads to a modified FF. By doing this, the OW-o repulsion decreases, which allows the $\mathrm{D}_{2} \mathrm{O}$ molecules to approach the framework more closely and establish stronger D-bonds with the carbonyl groups.

The effects of modifying the OW-o LJ potential can be directly seen in the differences between the radial distribution functions (RDFs) calculated from MD simulations carried out with the original and modified FFs (Fig. 3) as well as in the average water-MOF D-bond length in Table 1. In the original FF, the first peak in the OW-OW RDF is located at 2.8 $\AA$ (black), while the first peak in the $\mathrm{OW}-\mathrm{O}$ RDF is at $3.1 \AA$ (gray), suggesting that the $\mathrm{D}_{2} \mathrm{O}$ molecules approach each other closer than $\mathrm{D}_{2} \mathrm{O}$ molecules can approach the MOF atoms. On the other hand, the first peak in the OW-OW RDF remains at $2.8 \AA$ (royal blue) but the first peak in the OW-o RDF moves to $2.55 \AA$ (light blue) with the modified FF. This indicates that, while the interaction between $\mathrm{D}_{2} \mathrm{O}$ molecules is not affected by changes in 


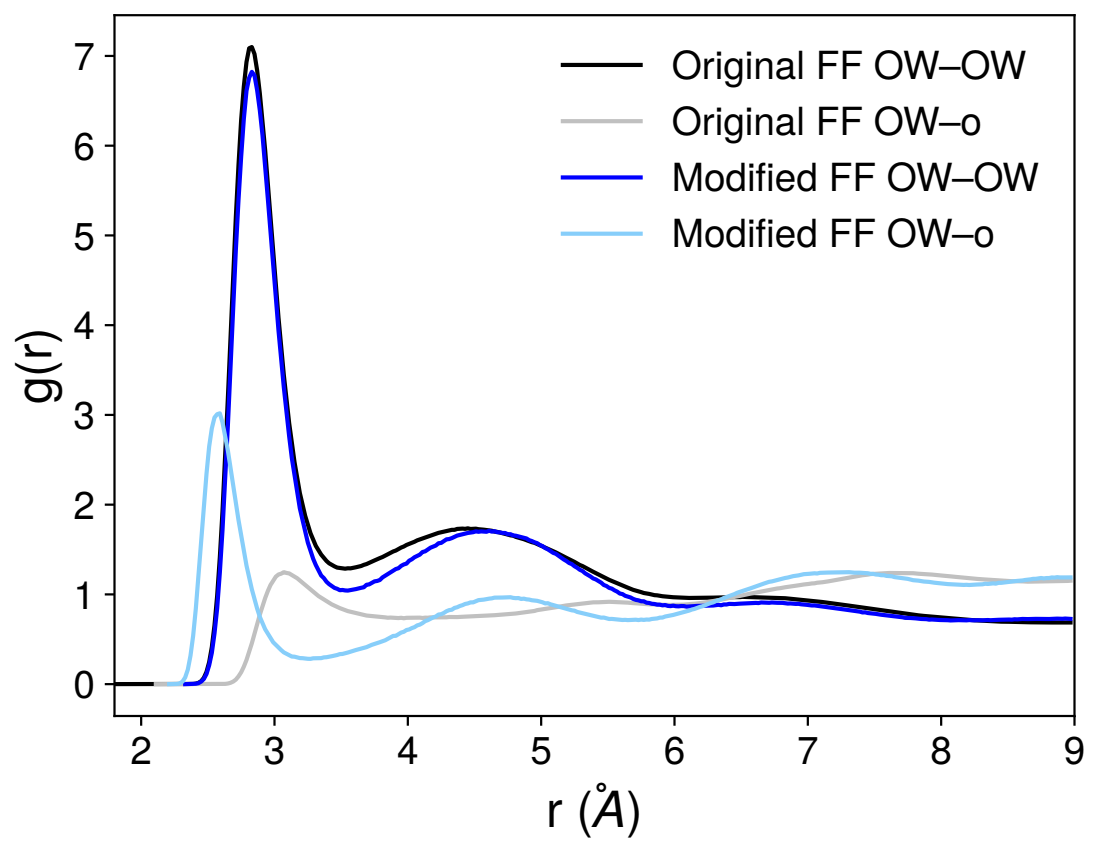

Figure 3: OW-OW (dark colors) and OW-o (light colors) RDFs of water confined in ZIF-90 in the original force field (black colors) and the modified force field (blue colors).

the framework- $\mathrm{D}_{2} \mathrm{O}$ interactions, the modified FF leads to $\mathrm{D}_{2} \mathrm{O}$ molecules forming shorter and stronger D-bonds with the framework than in the original FF (Table 1). It should be noted that other modifications of the original FF were also investigated and were found to significantly affect the $\mathrm{D}_{2} \mathrm{O}$ structure in the ZIF-90 pores, leading to large deviations in both RDFs and IR spectra as shown in the Supporting Information (Figs. S10, S11, and S12).

The influence of modifying the OW-o LJ potential is clearly seen in the IR spectra calculated from MD and CMD simulations which are shown in Fig. 4A and Fig. 4B, respectively. Decreasing $\sigma_{O W-o}$ by $10 \%$ and decreasing $\epsilon_{O W-o}$ by $20 \%$ in the modified $\mathrm{FF}$ redistributes the IR intensity between $\sim 2500-2550 \mathrm{~cm}^{-1}$. Specifically, the shorter water-MOF D-bonds in the modified FF move the IR intensity from the high-frequency range to the mid-frequency range of the OD-stretch lineshape, leaving the low-frequency peak of the strongest D-bonds between water molecules unchanged. With the modified FF, the CMD simulation closely reproduces the intensity of the two main peaks of the experimental spectrum at $\sim 2550$ and 

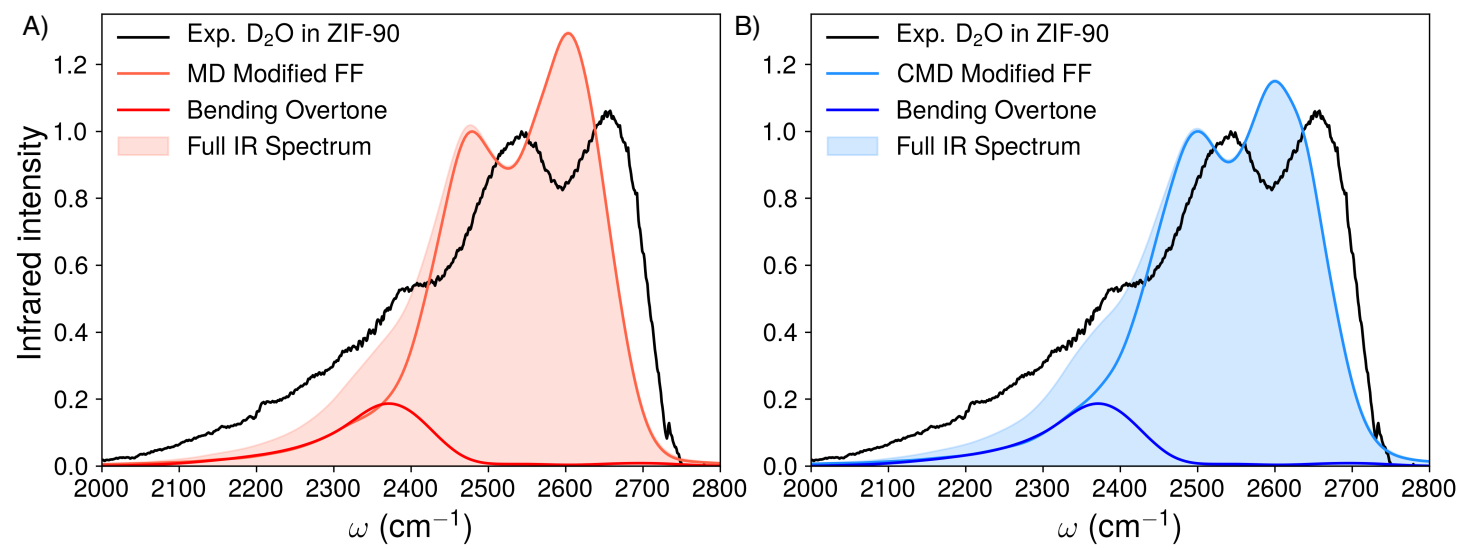

Figure 4: A) IR spectra of water in ZIF-90 at $40 \% \mathrm{RH}$ from experiment (black), MD at $40 \%$ $\mathrm{RH}$ for the modified FF (pink), Fermi resonance contribution (red), and MD at $40 \% \mathrm{RH}$ with added Fermi resonance contribution for the modified FF (shaded pink). B) IR spectra of water in ZIF-90 at 40\% RH from experiment (black), CMD at $40 \% \mathrm{RH}$ for the modified FF (light blue), Fermi resonance contribution (blue), and CMD at $40 \% \mathrm{RH}$ with added Fermi resonance contribution for the modified FF (shaded light blue). All MD spectra are red-shifted by $137 \mathrm{~cm}^{-1}$, CMD spectra are red-shifted by $57 \mathrm{~cm}^{-1}$, and DOD overtones are blue-shifted by $24 \mathrm{~cm}^{-1}$. All spectra are normalized to a value of one at the lower frequency peak.

$\sim 2650 \mathrm{~cm}^{-1}$. Furthermore, adding the Fermi resonance contributions increases the intensity on the red side of both the MD and CMD OD-stretch lineshapes, which makes the CMD lineshape reproduce the corresponding experimental lineshape nearly quantitatively. As seen in Table 1, simulations with the modified FF also lead to slightly longer OW-OW D-bond which results in the loss of some IR intensity from the lower-frequency peak at $\sim 2450 \mathrm{~cm}^{-1}$ (Fig. 4).

To provide further insights into the structural arrangements of the $\mathrm{D}_{2} \mathrm{O}$ molecules in the ZIF-90 pores in connection with the OD-stretch lineshape, Fig. 5 shows the distribution of different D-bond topologies for both bulk $\mathrm{D}_{2} \mathrm{O}$ and $\mathrm{D}_{2} \mathrm{O}$ in ZIF-90. The majority of bulk $\mathrm{D}_{2} \mathrm{O}$ molecules donate and accept at least one D-bond, with the varying number of donors and acceptors resulting in the broad OD-stretch band. On the other hand, the MD simulations of $\mathrm{D}_{2} \mathrm{O}$ in ZIF-90 carried out with the original $\mathrm{FF}$ indicate that, among $\mathrm{D}_{2} \mathrm{O}$ molecules not involved in D-bonding with the framework (Fig. 5A), a relatively smaller fraction donates 
and accepts at least one D-bond compared to bulk $\mathrm{D}_{2} \mathrm{O}$. When the $\mathrm{D}_{2} \mathrm{O}$ molecules donate one D-bond to the framework (Fig. 5B), the fraction of $\mathrm{D}_{2} \mathrm{O}$ molecules that are involved in D-bonding with themselves is significantly smaller than in bulk $\mathrm{D}_{2} \mathrm{O}$. Therefore, due to the overall smaller number of D-bond donors and acceptors, the vibrations of the $\mathrm{D}_{2} \mathrm{O}$ molecules confined in ZIF-90 retain some character of the symmetric and asymmetric stretches of gas-phase $\mathrm{D}_{2} \mathrm{O}$, which results in the two distinct peaks found in the IR spectra.

The comparison between the D-bonding topologies obtained with the original and modified FFs indicates that the latter leads to an overall decrease in the number of $\mathrm{D}_{2} \mathrm{O}$ molecules that are D-bonded with themselves when the $\mathrm{D}_{2} \mathrm{O}$ molecules are not involved in D-bonding with the framework (Fig. 5A). This is accompanied by an overall increase of $\mathrm{D}_{2} \mathrm{O}$ molecules that donate D-bonds to the carbonyl groups of the framework, whose fraction increases from $25 \%$ in the MD simulations with the original $\mathrm{FF}$ to $40 \%$ in the MD simulations with the modified $\mathrm{FF}$, resulting in a more tetrahedral arrangement of the $\mathrm{D}_{2} \mathrm{O}$ molecules in the ZIF90 pores (Fig. S13). Given the differences in strength between the stronger $\mathrm{D}_{2} \mathrm{O}-\mathrm{D}_{2} \mathrm{O}$ and weaker $\mathrm{D}_{2} \mathrm{O}$-carbonyl D-bonds, ${ }^{110}$ this analysis demonstrates that the rearrangements of the D-bonding network predicted by the MD simulations with the modified FF are responsible for the redistribution of the IR intensity in the $\sim 2500-2550 \mathrm{~cm}^{-1}$ frequency range of the simulated OD-stretch lineshapeshown in Fig. 4.

A small red shift between the experimental and simulated OD-stretch lineshapes still remains after modifying the FF and including the Fermi resonances (Fig. 4). In this context, it should be reminded that the intrinsic shifts applied the simulated lineshapes are based on the analysis of the IR spectrum of bulk $\mathrm{D}_{2} \mathrm{O}$. As highlighted in Fig. $1 \mathrm{C}$, there is a $\sim 40 \mathrm{~cm}^{-1}$ blue shift in the experimental spectra of $\mathrm{D}_{2} \mathrm{O}$ confined in ZIF-90 relative to bulk $\mathrm{D}_{2} \mathrm{O}$. This blue shift accounts for the small differences that remain in the higher-frequency portion of the lineshape. Although the level of agreement with the experimental OD-stretch lineshape is nearly quantitative, the simulated lineshapes display slightly lower intensities between $2000 \mathrm{~cm}^{-1}$ and $2400 \mathrm{~cm}^{-1}$. As discussed above, this frequency range is primarily associated 

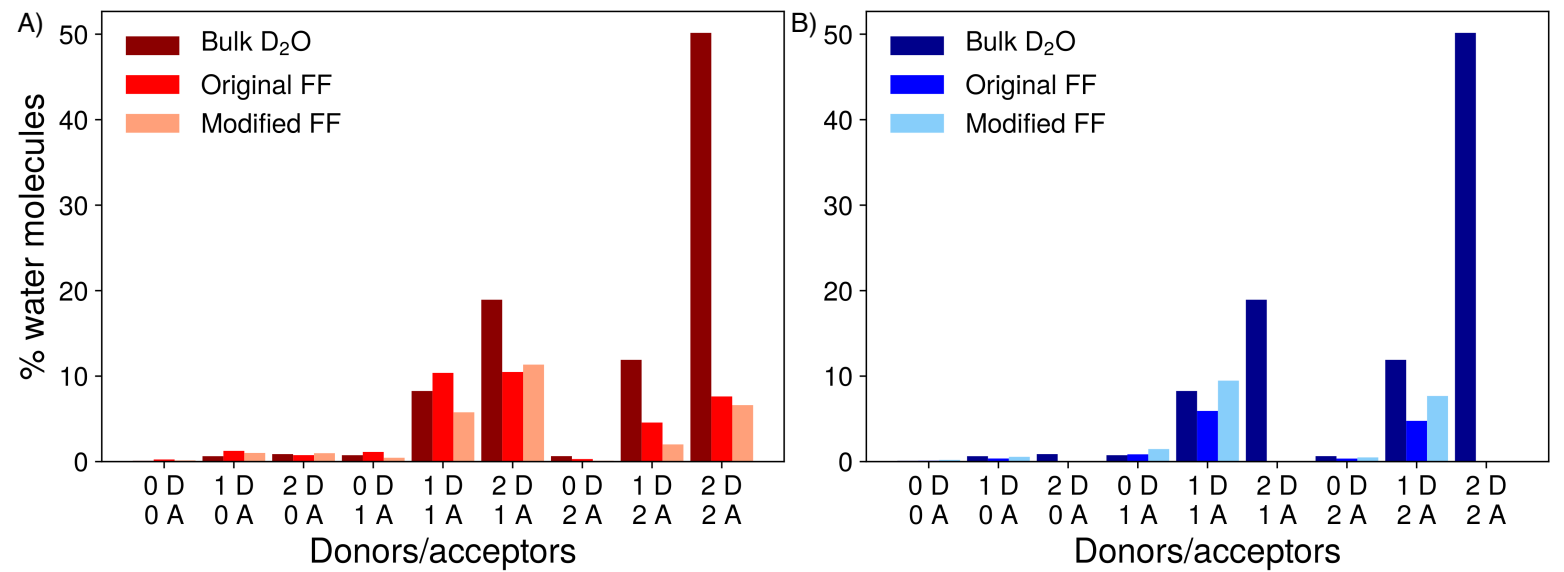

Figure 5: A) Donating (D) and accepting (A) D-bond distribution of water molecules when there are zero D-bonds donated to the MOF. D-bond distributions are shown for bulk $\mathrm{D}_{2} \mathrm{O}$ (dark red), the original FF (red), and the modified FF (light red). D-bonds are plotted as a percent of the total number of water molecules in each simulation. B) Donating (D) and accepting (A) D-bond distribution of water molecules when there is one D-bond donated to the MOF. D-bond distributions are shown for bulk $\mathrm{D}_{2} \mathrm{O}$ (dark blue), the original FF (blue), and the modified FF (light blue). D-bonds are plotted as a percent of the total number of water molecules in each simulation.

with Fermi resonances. While this is the first study where Fermi resonance contributions are included in simulations of the IR spectra of water in MOFs, state-of-the-art calculations currently only allow for determining these contributions from simulations of relatively small gas-phase water clusters. This approximation is likely responsible for the remaining differences between experimental and simulated lineshapes in the $2000-2400 \mathrm{~cm}^{-1}$ frequency region.

\section{Conclusions}

In this study, we have characterized the structure of $\mathrm{D}_{2} \mathrm{O}$ adsorbed in ZIF-90 as a function of relative humidity by integrating experimental and computational IR spectroscopy. Due to the confining environment provided by the framework, the $\mathrm{D}_{2} \mathrm{O}$ molecules are found to establish longer and relatively weaker D-bonds that manifest in an OD-stretch band that is blue shifted compared to bulk. A systematic analysis of the OD-stretch band, carried out 
using both (classical) MD and (quantum) CMD simulations demonstrates the importance of explicitly taking into account both Fermi resonances and nuclear quantum effects. Our simulations indicate that the $\mathrm{D}_{2} \mathrm{O}$ molecules preferentially interact with the carbonyl groups of the framework. However, it is found that "off-the-shelf" force fields commonly used in MD simulations of MOFs are unable to correctly represent the strength of the framework-water interactions. Guided by direct comparisons with the experimental OD-stretch lineshape, we have demonstrated that it is possible to use the comparison between experimental and simulated IR spectra to guide the refinement of the force field parameterization representing the framework-water interactions. This "reverse engineering" process has led to a refined set of Lennard-Jones parameters describing the interactions between the oxygen atoms of the water molecules and the carbonyl groups of the framework, which results in nearly quantitative agreement between the experimental and simulated OD-stretch lineshapes and, in turn, allows for an accurate, molecular-level characterization of the structural arrangements of the $\mathrm{D}_{2} \mathrm{O}$ molecules inside the ZIF-90 pores. Our results suggest that the integration of experimental and computational vibrational spectroscopy can play an important role in characterizing the molecular properties of water adsorbed in MOFs and identifying key framework-water interactions, which is critical for the design of efficient MOF-based materials for water harvesting.

\section{Associated Content}

Experimental PXRD, SEM, and humidity generator; all force field parameters for ZIF-90; additional comparisons of experimental and simulated IR spectra; structural and dynamical properties of water confined in ZIF-90. 


\section{Acknowledgments}

This research was supported by the Department of Energy under contract DE-SC0019333 and the National Science Foundation through grant No. CHE-1704063. All simulations used resources of the National Energy Research Scientific Computing Center (NERSC), which is supported by the Office of Science of the U.S. Department of Energy under contract DEAC02-05CH11231, the Extreme Science and Engineering Discovery Environment (XSEDE), which is supported by the National Science Foundation through award No. ACI-1548562, as well as the Triton Shared Computing Cluster (TSCC) at the San Diego Supercomputer Center (SDSC). M.K. is supported by the Department of Defense (DoD) through the National

Defense Science and Engineering Graduate (NDSEG) Fellowship and is the recipient of an Achievement Rewards for College Scientists (ARCS) Foundation Fellowship.

\section{References}

(1) Hong, B. H.; Lee, J. Y.; Lee, C.-W.; Kim, J. C.; Bae, S. C.; Kim, K. S. Self-Assembled Arrays of Organic Nanotubes with Infinitely Long One-Dimensional H-Bond Chains. J. Am. Chem. Soc. 2001, 123, 10748-10749.

(2) Amoli, V.; Kim, J. S.; Jee, E.; Chung, Y. S.; Kim, S. Y.; Koo, J.; Choi, H.; Kim, Y.; Kim, D. H. A Bioinspired Hydrogen Bond-Triggered Ultrasensitive Ionic Mechanoreceptor Skin. Nat. Commun. 2019, 10, 1-13.

(3) Mehra, N.; Jeske, M.; Yang, X.; Gu, J.; Kashfipour, M. A.; Li, Y.; Baughman, J. A.; Zhu, J. Hydrogen-Bond Driven Self-Assembly of Two-Dimensional Supramolecular Melamine-Cyanuric Acid Crystals and its Self-Alignment in Polymer Composites for Enhanced Thermal Conduction. ACS Appl. Polym. Mater. 2019, 1, 1291-1300.

(4) Gong, Y.; Xu, Y.; Zhou, Y.; Li, C.; Liu, X.; Niu, L.; Huang, Y.; Zhang, X.; 
Sun, C. Q. Hydrogen Bond Network Relaxation Resolved by Alcohol Hydration (Methanol, Ethanol, and Glycerol). J. Raman Spectrosc. 2017, 48, 393-398.

(5) Arayachukiat, S.; Kongtes, C.; Barthel, A.; Vummaleti, S. V.; Poater, A.; Wannakao, S.; Cavallo, L.; D'Elia, V. Ascorbic Acid as a Bifunctional Hydrogen Bond Donor for the Synthesis of Cyclic Carbonates from $\mathrm{CO}_{2}$ under Ambient Conditions. ACS Sustain. Chem. Eng. 2017, 5, 6392-6397.

(6) Munoz-Santiburcio, D.; Marx, D. Chemistry in Nanoconfined Water. Chem. Sci. 2017, $8,3444-3452$.

(7) Wang, H.; Wagner, J. C.; Chen, W.; Wang, C.; Xiong, W. Spatially Dependent HBond Dynamics at Interfaces of Water/Biomimetic Self-Assembled Lattice Materials. Proc. Natl. Acad. Sci. 2020, 117, 23385-23392.

(8) Li, J.; Chi, Z.; Qin, R.; Yan, L.; Lin, X.; Hu, M.; Shan, G.; Chen, H.; Weng, Y.X. Hydrogen Bond Interaction Promotes Flash Energy Transport at MXene-Solvent Interface. J. Phys. Chem. C 2020, 124, 10306-10314.

(9) He, F.; Wang, Q.; Hu, C.; He, W.; Luo, X.; Huang, L.; Gao, D.; Bi, J.; Wang, X.; Zou, G. Centrosymmetric (NH4) 2SbCl (SO4) 2 and Non-Centrosymmetric (NH4) SbCl2 (SO4): Synergistic Effect of Hydrogen-Bonding Interactions and Lone-Pair Cations on the Framework Structures and Macroscopic Centricities. Cryst. Growth Des. 2018, 18, 6239-6247.

(10) Shao, C.; Chang, H.; Wang, M.; Xu, F.; Yang, J. High-Strength, Tough, and SelfHealing Nanocomposite Physical Hydrogels Based on the Synergistic Effects of Dynamic Hydrogen Bond and Dual Coordination Bonds. ACS Appl. Mater. Interfaces 2017, 9, 28305-28318.

(11) Wang, H.; Chen, W.; Wagner, J. C.; Xiong, W. Local Ordering of Lattice Self- 
Assembled SDS@ 2 $\beta$-CD Materials and Adsorbed Water Revealed by Vibrational Sum Frequency Generation Microscope. J. Phys. Chem. B 2019, 123, 6212-6221.

(12) Knight, A. W.; Kalugin, N. G.; Coker, E.; Ilgen, A. G. Water Properties under NanoScale Confinement. Sci. Rep. 2019, 9, 1-12.

(13) Chakraborty, S.; Kumar, H.; Dasgupta, C.; Maiti, P. K. Confined Water: Structure, Dynamics, and Thermodynamics. Acc. Chem. Res. 2017, 50, 2139-2146.

(14) Rasaiah, J. C.; Garde, S.; Hummer, G. Water in Nonpolar Confinement: From Nanotubes to Proteins and Beyond. Annu. Rev. Phys. Chem. 2008, 59, 713-740.

(15) Hummer, G.; Rasaiah, J. C.; Noworyta, J. P. Water Conduction through the Hydrophobic Channel of a Carbon Nanotube. Nature 2001, 414, 188-190.

(16) Mukherjee, B.; Maiti, P. K.; Dasgupta, C.; Sood, A. Jump Reorientation of Water Molecules Confined in Narrow Carbon Nanotubes. J. Phys. Chem. B 2009, 113, $10322-10330$.

(17) Barati Farimani, A.; Aluru, N. R. Spatial Diffusion of Water in Carbon Nanotubes: from Fickian to Ballistic Motion. J. Phys. Chem. B 2011, 115, 12145-12149.

(18) Crupi, V.; Longo, F.; Majolino, D.; Venuti, V. Vibrational Properties of Water Molecules Adsorbed in Different Zeolitic Frameworks. J. Phys. Condens. Matter 2006, $18,3563$.

(19) Yamada, S. A.; Shin, J. Y.; Thompson, W. H.; Fayer, M. D. Water Dynamics in Nanoporous Silica: uUtrafast Vibrational Spectroscopy and Molecular Dynamics Simulations. J. Phys. Chem. C 2019, 123, 5790-5803.

(20) Tan, H.-S.; Piletic, I. R.; Fayer, M. Orientational Dynamics of Water Confined on a Nanometer Length Scale in Reverse Micelles. J. Chem. Phys. 2005, 122, 174501. 
(21) Scodinu, A.; Fourkas, J. T. Comparison of the Orientational Dynamics of Water Confined in Hydrophobic and Hydrophilic Nanopores. J. Phys. Chem. B 2002, 106, $10292-10295$.

(22) Furukawa, H.; Cordova, K. E.; O'Keeffe, M.; Yaghi, O. M. The Chemistry and Applications of Metal-Organic Frameworks. Science 2013, 341.

(23) Canivet, J.; Fateeva, A.; Guo, Y.; Coasne, B.; Farrusseng, D. Water Adsorption in MOFs: Fundamentals and Applications. Chem. Soc. Rev. 2014, 43, 5594-5617.

(24) Liu, X.; Wang, X.; Kapteijn, F. Water and Metal-Organic Frameworks: From Interaction toward Utilization. Chem. Rev. 2020, 120, 8303-8377.

(25) Sumida, K.; Rogow, D. L.; Mason, J. A.; McDonald, T. M.; Bloch, E. D.; Herm, Z. R.; Bae, T.-H.; Long, J. R. Carbon Dioxide Capture in Metal-Organic Frameworks. Chem. Rev. 2012, 112, 724-781.

(26) Getman, R. B.; Bae, Y.-S.; Wilmer, C. E.; Snurr, R. Q. Review and Analysis of Molecular Simulations of Methane, Hydrogen, and Acetylene Storage in Metal-Organic Frameworks. Chem. Rev. 2012, 112, 703-723.

(27) Qian, Q.; Asinger, P. A.; Lee, M. J.; Han, G.; Mizrahi Rodriguez, K.; Lin, S.; Benedetti, F. M.; Wu, A. X.; Chi, W. S.; Smith, Z. P. MOF-Based Membranes for Gas Separations. Chem. Rev. 2020, 120, 8161-8266.

(28) Rojas, S.; Horcajada, P. Metal-Organic Frameworks for the Removal of Emerging Organic Contaminants in Water. Chem. Rev. 2020, 120, 8378-8415.

(29) Bavykina, A.; Kolobov, N.; Khan, I. S.; Bau, J. A.; Ramirez, A.; Gascon, J. MetalOrganic Frameworks in Heterogeneous Catalysis: Recent Progress, New Trends, and Future Perspectives. Chem. Rev. 2020, 120, 8468-8535. 
(30) Islamoglu, T.; Chen, Z.; Wasson, M. C.; Buru, C. T.; Kirlikovali, K. O.; Afrin, U.; Mian, M. R.; Farha, O. K. Metal-Organic Frameworks Against Toxic Chemicals. Chem. Rev. 2020, 120, 8130-8160.

(31) Lim, D.-W.; Kitagawa, H. Proton Transport in Metal-Organic Frameworks. Chem. Rev. 2020, 120, 8416-8467.

(32) Allendorf, M. D.; Dong, R.; Feng, X.; Kaskel, S.; Matoga, D.; Stavila, V. Electronic Devices Using Open Framework Materials. Chem. Rev. 2020, 120, 8581-8640.

(33) Thorarinsdottir, A. E.; Harris, T. D. Metal-Organic Framework Magnets. Chem. Rev. 2020, 120, 8716-8789.

(34) Fathieh, F.; Kalmutzki, M. J.; Kapustin, E. A.; Waller, P. J.; Yang, J.; Yaghi, O. M. Practical Water Production from Desert Air. Sci. Adv. 2018, 4, eaat3198.

(35) Hanikel, N.; Prévot, M. S.; Fathieh, F.; Kapustin, E. A.; Lyu, H.; Wang, H.; Diercks, N. J.; Glover, T. G.; Yaghi, O. M. Rapid Cycling and Exceptional Yield in a Metal-Organic Framework Water Harvester. ACS Cent. Sci. 2019, 5, 1699-1706.

(36) Rieth, A. J.; Yang, S.; Wang, E. N.; Dincă, M. Record Atmospheric Fresh Water Capture and Heat Transfer With a Material Operating at the Water Uptake Reversibility Limit. ACS Cent. Sci. 2017, 3, 668-672.

(37) Rieth, A. J.; Wright, A. M.; Skorupskii, G.; Mancuso, J. L.; Hendon, C. H.; Dincă, M. Record-Setting Sorbents for Reversible Water Uptake by Systematic Anion Exchanges in Metal-Organic Frameworks. J. Am. Chem. Soc. 2019, 141, 13858-13866.

(38) Chen, Z.; Li, P.; Zhang, X.; Li, P.; Wasson, M. C.; Islamoglu, T.; Stoddart, J. F.; Farha, O. K. Reticular Access to Highly Porous acs-MOFs with Rigid Trigonal Prismatic Linkers for Water Sorption. J. Am. Chem. Soc. 2019, 141, 2900-2905. 
(39) Bakker, H.; Skinner, J. Vibrational Spectroscopy as a Probe of Structure and Dynamics in Liquid Water. Chem. Rev. 2010, 110, 1498-1517.

(40) Salazar, J.; Weber, G.; Simon, J.; Bezverkhyy, I.; Bellat, J. Characterization of Adsorbed Water in MIL-53 (Al) by FTIR Spectroscopy and Ab-Initio Calculations. J. Chem. Phys. 2015, 142, 124702.

(41) Rieth, A. J.; Hunter, K. M.; Dincă, M.; Paesani, F. Hydrogen Bonding Structure of Confined Water Templated by a Metal-Organic Framework with Open Metal Sites. Nat. Commun. 2019, 10, 1-7.

(42) Wang, J.; Wolf, R. M.; Caldwell, J. W.; Kollman, P. A.; Case, D. A. Development and Testing of a General Amber Force Field. J. Comput. Chem. 2004, 25, 1157-1174.

(43) Rappé, A. K.; Casewit, C. J.; Colwell, K.; Goddard III, W. A.; Skiff, W. M. UFF, a Full Periodic Table Force Field for Molecular Mechanics and Molecular Dynamics Simulations. J. Am. Chem. Soc. 1992, 114, 10024-10035.

(44) Bureekaew, S.; Amirjalayer, S.; Tafipolsky, M.; Spickermann, C.; Roy, T. K.; Schmid, R. MOF-FF-A Flexible First-Principles Derived Force Field for MetalOrganic Frameworks. Phys. Status Solidi B 2013, 250, 1128-1141.

(45) Addicoat, M. A.; Vankova, N.; Akter, I. F.; Heine, T. Extension of the Universal Force Field to Metal-Organic Frameworks. J. Chem. Theory Comput. 2014, 10, 880-891.

(46) Vanduyfhuys, L.; Vandenbrande, S.; Verstraelen, T.; Schmid, R.; Waroquier, M.; Van Speybroeck, V. QuickFF: A Program for a Quick and Easy Derivation of Force Fields for Metal-Organic Frameworks from Ab Initio Input. J. Comput. Chem. 2015, 36, 1015-1027.

(47) Coupry, D. E.; Addicoat, M. A.; Heine, T. Extension of the Universal Force Field for Metal-Organic Frameworks. J. Chem. Theory Comput. 2016, 12, 5215-5225. 
(48) Weng, T.; Schmidt, J. Flexible and Transferable Ab Initio Force Field for Zeolitic Imidazolate Frameworks: ZIF-FF. J. Phys. Chem. A 2019, 123, 3000-3012.

(49) Cirera, J.; Sung, J. C.; Howland, P. B.; Paesani, F. The Effects of Electronic Polarization on Water Adsorption in Metal-Organic Frameworks: $\mathrm{H}_{2} \mathrm{O}$ in MIL-53 (Cr). $J$. Chem. Phys. 2012, 137, 054704.

(50) McDaniel, J. G.; Li, S.; Tylianakis, E.; Snurr, R. Q.; Schmidt, J. Evaluation of Force Field Performance for High-Throughput Screening of Gas Uptake in Metal-Organic Frameworks. J. Phys. Chem. C 2015, 119, 3143-3152.

(51) Becker, T. M.; Luna-Triguero, A.; Vicent-Luna, J. M.; Lin, L.-C.; Dubbeldam, D.; Calero, S.; Vlugt, T. J. Potential of Polarizable Force Fields for Predicting the Separation Performance of Small Hydrocarbons in M-MOF-74. Phys. Chem. Chem. Phys. 2018, 20, 28848-28859.

(52) Becker, T. M.; Lin, L.-C.; Dubbeldam, D.; Vlugt, T. J. Polarizable Force Field for $\mathrm{CO}_{2}$ in M-MOF-74 Derived from Quantum Mechanics. J. Phys. Chem. C 2018, 122, 24488-24498.

(53) Suepaul, S.; Forrest, K. A.; Pham, T.; Space, B. Investigating the Effects of Linker Extension on H2 Sorption in the Rht-Metal-Organic Framework Nu-111 by Molecular Simulations. Cryst. Growth Des. 2018, 18, 7599-7610.

(54) Hogan, A.; Space, B. Next-Generation Accurate, Transferable, and Polarizable Potentials for Material Simulations. J. Chem. Theory Comput. 2020, 16, 7632-7644.

(55) Nilsson, A.; Pettersson, L. G. The Structural Origin of Anomalous Properties of Liquid Water. Nat. Commun. 2015, 6, 1-11.

(56) Gallo, P.; Amann-Winkel, K.; Angell, C. A.; Anisimov, M. A.; Caupin, F.; 
Chakravarty, C.; Lascaris, E.; Loerting, T.; Panagiotopoulos, A. Z.; Russo, J., et al. Water: A Tale of Two Liquids. Chem. Rev. 2016, 116, 7463-7500.

(57) Cerveny, S.; Mallamace, F.; Swenson, J.; Vogel, M.; Xu, L. Confined Water as Model of Supercooled Water. Chem. Rev. 2016, 116, 7608-7625.

(58) Ortiz, A. U.; Freitas, A. P.; Boutin, A.; Fuchs, A. H.; Coudert, F.-X. What Makes Zeolitic Imidazolate Frameworks Hydrophobic or Hydrophilic? The Impact of Geometry and Functionalization on Water Adsorption. Phys. Chem. Chem. Phys. 2014, 16, 9940-9949.

(59) Calero, S.; Gómez-Álvarez, P. Underlying Adsorption Mechanisms of Water in Hydrophobic and Hydrophilic Zeolite Imidazolate Frameworks: ZIF-71 and ZIF-90. J. Phys. Chem. C 2015, 119, 23774-23780.

(60) Gao, M.; Wang, J.; Rong, Z.; Shi, Q.; Dong, J. A Combined ExperimentalComputational Investigation on Water Adsorption in Various ZIFs with the SOD and RHO Topologies. RSC Adv. 2018, 8, 39627-39634.

(61) Huang, A.; Dou, W.; Caro, J. Steam-Stable Zeolitic Imidazolate Framework ZIF90 Membrane with Hydrogen Selectivity through Covalent Functionalization. J. Am. Chem. Soc. 2010, 132, 15562-15564.

(62) Yang, T.; Chung, T.-S. Room-Temperature Synthesis of ZIF-90 Nanocrystals and the Derived Nano-Composite Membranes for Hydrogen Separation. J. Mater. Chem. A 2013, 1, 6081-6090.

(63) Kresse, G.; Hafner, J. Ab Initio Molecular Dynamics for Liquid Metals. Phys. Rev. B 1993, 47, 558 .

(64) Kresse, G.; Hafner, J. Ab Initio Molecular-Dynamics Simulation of the Liquid-MetalAmorphous-Semiconductor Transition in Germanium. Phys. Rev. B 1994, 49, 14251. 
(65) Kresse, G.; Furthmüller, J. Efficiency of Ab-Initio Total Energy Calculations for Metals and Semiconductors Using a Plane-Wave Basis Set. Comput. Mater. Sci. 1996, 6, 1550.

(66) Kresse, G.; Furthmüller, J. Efficient Iterative Schemes for Ab Initio Total-Energy Calculations Using a Plane-Wave Basis Set. Phys. Rev. B 1996, 54, 11169.

(67) Perdew, J. P.; Burke, K.; Ernzerhof, M. Generalized Gradient Approximation Made Simple. Phys. Rev. Lett. 1996, 77, 3865.

(68) Grimme, S.; Antony, J.; Ehrlich, S.; Krieg, H. A Consistent and Accurate Ab Initio Parametrization of Density Functional Dispersion Correction (DFT-D) for the 94 Elements H-Pu. J. Chem. Phys. 2010, 132, 154104.

(69) Blöchl, P. E. Projector Augmented-Wave Method. Phys. Rev. B 1994, 50, 17953.

(70) Kresse, G.; Joubert, D. From Ultrasoft Pseudopotentials to the Projector AugmentedWave Method. Phys. Rev. B 1999, 59, 1758.

(71) Manz, T. A.; Sholl, D. S. Improved Atoms-in-Molecule Charge Partitioning Functional for Simultaneously Reproducing the Electrostatic Potential and Chemical States in Periodic and Nonperiodic Materials. J. Chem. Theory Comput. 2012, 8, 2844-2867.

(72) Babin, V.; Leforestier, C.; Paesani, F. Development of a "First Principles" Water Potential with Flexible Monomers: Dimer Potential Energy Surface, VRT Spectrum, and Second Virial Coefficient. J. Chem. Theory Comput. 2013, 9, 5395-5403.

(73) Babin, V.; Medders, G. R.; Paesani, F. Development of a "First Principles" Water Potential with Flexible Monomers. II: Trimer Potential Energy Surface, Third Virial Coefficient, and Small Clusters. J. Chem. Theory Comput. 2014, 10, 1599-1607.

(74) Medders, G. R.; Babin, V.; Paesani, F. Development of a "First-Principles" Water 
Potential with Flexible Monomers. III. Liquid Phase Properties. J. Chem. Theory Comput. 2014, 10, 2906-2910.

(75) Paesani, F. Getting the Right Answers for the Right Reasons: Toward Predictive Molecular Simulations of Water with Many-Body Potential Energy Functions. Acc. Chem. Res. 2016, 49, 1844-1851.

(76) Reddy, S. K.; Straight, S. C.; Bajaj, P.; Huy Pham, C.; Riera, M.; Moberg, D. R.; Morales, M. A.; Knight, C.; Götz, A. W.; Paesani, F. On the Accuracy of the MB-pol Many-Body Potential for Water: Interaction Energies, Vibrational Frequencies, and Classical Thermodynamic and Dynamical Properties from Clusters to Liquid Water and Ice. J. Chem. Phys. 2016, 145, 194504.

(77) Brown, S. E.; Götz, A. W.; Cheng, X.; Steele, R. P.; Mandelshtam, V. A.; Paesani, F. Monitoring Water Clusters "Melt" through Vibrational Spectroscopy. J. Am. Chem. Soc. 2017, 139, 7082-7088.

(78) Samala, N. R.; Agmon, N. Temperature Dependence of Intramolecular Vibrational Bands in Small Water Clusters. J. Phys. Chem. B 2019, 123, 9428-9442.

(79) Medders, G. R.; Paesani, F. Infrared and Raman Spectroscopy of Liquid Water through "First-Principles" Many-Body Molecular Dynamics. J. Chem. Theory Comput. 2015, 11, 1145-1154.

(80) Straight, S. C.; Paesani, F. Exploring Electrostatic Effects on the Hydrogen Bond Network of Liquid Water through Many-Body Molecular Dynamics. J. Phys. Chem. B 2016, 120, 8539-8546.

(81) Reddy, S. K.; Moberg, D. R.; Straight, S. C.; Paesani, F. Temperature-Dependent Vibrational Spectra and Structure of Liquid Water from Classical and Quantum Simulations with the MB-pol Potential Energy Function. J. Chem. Phys. 2017, 147, 244504. 
(82) Hunter, K. M.; Shakib, F. A.; Paesani, F. Disentangling Coupling Effects in the Infrared Spectra of Liquid Water. J. Phys. Chem. B 2018, 122, 10754-10761.

(83) Medders, G. R.; Paesani, F. Dissecting the Molecular Structure of the Air/Water Interface from Quantum Simulations of the Sum-Frequency Generation Spectrum. J. Am. Chem. Soc. 2016, 138, 3912-3919.

(84) Moberg, D. R.; Straight, S. C.; Paesani, F. Temperature Dependence of the Air/Water Interface Revealed by Polarization Sensitive Sum-Frequency Generation Spectroscopy. J. Phys. Chem. B 2018, 122, 4356-4365.

(85) Moberg, D. R.; Straight, S. C.; Knight, C.; Paesani, F. Molecular Origin of the Vibrational Structure of Ice Ih. J. Phys. Chem. Lett. 2017, 8, 2579-2583.

(86) Moberg, D. R.; Sharp, P. J.; Paesani, F. Molecular-Level Interpretation of Vibrational Spectra of Ordered Ice Phases. J. Phys. Chem. B 2018, 122, 10572-10581.

(87) Moberg, D. R.; Becker, D.; Dierking, C. W.; Zurheide, F.; Bandow, B.; Buck, U.; Hudait, A.; Molinero, V.; Paesani, F.; Zeuch, T. The End of Ice I. Proc. Natl. Acad. Sci. 2019, 116, 24413-24419.

(88) Riera, M.; Yeh, E. P.; Paesani, F. Data-Driven Many-Body Models for Molecular Fluids: $\mathrm{CO}_{2} / \mathrm{H}_{2} \mathrm{O}$ Mixtures as a Case Study. J. Chem. Theory Comput. 2020, 16, $2246-2257$.

(89) Riera, M.; Hirales, A.; Ghosh, R.; Paesani, F. Data-Driven Many-Body Models with Chemical Accuracy for $\mathrm{CH}_{4} / \mathrm{H}_{2} \mathrm{O}$ Mixtures. J. Phys. Chem. B 2020, 124, 1120711221.

(90) Abascal, J. L.; Vega, C. A General Purpose Model for the Condensed Phases of Water: TIP4P/2005. J. Chem. Phys. 2005, 123, 234505. 
(91) Hudait, A.; Moberg, D. R.; Qiu, Y.; Odendahl, N.; Paesani, F.; Molinero, V. Preordering of water is not needed for ice recognition by hyperactive antifreeze proteins. Proc. Natl. Acad. Sci. U.S.A. 2018, 115, 8266-8271.

(92) Smith, W.; Forester, T. DL_POLY_2. 0: A General-Purpose Parallel Molecular Dynamics Simulation Package. J. Mol. Graph. 1996, 14, 136-141.

(93) Leach, A. R. Molecular Modelling: Principles and Applications; Pearson education, 2001.

(94) Cao, J.; Voth, G. A. The Formulation of Quantum Statistical Mechanics Based on the Feynman Path Centroid Density. I. Equilibrium Properties. J. Chem. Phys. 1994, $100,5093-5105$.

(95) Cao, J.; Voth, G. A. The Formulation of Quantum Statistical Mechanics Based on the Feynman Path Centroid Density. II. Dynamical Properties. J. Chem. Phys. 1994, $100,5106-5117$.

(96) Cao, J.; Voth, G. A. The Formulation of Quantum Statistical Mechanics Based on the Feynman Path Centroid Density. III. Phase Space Formalism and Analysis of Centroid Molecular Dynamics. J. Chem. Phys. 1994, 101, 6157-6167.

(97) Cao, J.; Voth, G. A. The Formulation of Quantum Statistical Mechanics Based on the Feynman Path Centroid Density. IV. Algorithms for Centroid Molecular Dynamics. J. Chem. Phys. 1994, 101, 6168-6183.

(98) Cao, J.; Voth, G. A. The Formulation of Quantum Statistical Mechanics Based on the Feynman Path Centroid Density. V. Quantum Instantaneous Normal Mode Theory of Liquids. J. Chem. Phys. 1994, 101, 6184-6192.

(99) Hone, T. D.; Rossky, P. J.; Voth, G. A. A Comparative Study of Imaginary Time Path Integral Based Methods for Quantum Dynamics. J. Chem. Phys. 2006, 124, 154103. 
(100) Paesani, F.; Voth, G. A. A Quantitative Assessment of the Accuracy of Centroid Molecular Dynamics for the Calculation of the Infrared Spectrum of Liquid Water. J. Chem. Phys. 2010, 132, 014105.

(101) Rossi, M.; Liu, H.; Paesani, F.; Bowman, J.; Ceriotti, M. Communication: On the consistency of approximate quantum dynamics simulation methods for vibrational spectra in the condensed phase. 2014.

(102) Kananenka, A. A.; Skinner, J. Fermi Resonance in OH-Stretch Vibrational Spectroscopy of Liquid Water and the Water Hexamer. J. Chem. Phys. 2018, 148, 244107.

(103) Wang, Y.; Bowman, J. M. Ab Initio Potential and Dipole Moment Surfaces for Water. II. Local-Monomer Calculations of the Infrared Spectra of Water Clusters. J. Chem. Phys. 2011, 134, 154510.

(104) Cheng, X.; Steele, R. P. Efficient Anharmonic Vibrational Spectroscopy for Large Molecules Using Local-Mode Coordinates. J. Chem. Phys. 2014, 141, 104105.

(105) Cheng, X.; Talbot, J. J.; Steele, R. P. Tuning Vibrational Mode Localization with Frequency Windowing. J. Chem. Phys. 2016, 145, 124112.

(106) Neese, F. The ORCA Program System. Wiley Interdiscip. Rev.: Comput. Mol. Sci. 2012, 2, 73-78.

(107) De Marco, L.; Ramasesha, K.; Tokmakoff, A. Experimental Evidence of Fermi Resonances in Isotopically Dilute Water from Ultrafast Broadband IR Spectroscopy. J. Phys. Chem. B 2013, 117, 15319-15327.

(108) McCoy, A. B. The Role of Electrical Anharmonicity in the Association Band in the Water Spectrum. J. Phys. Chem. B 2014, 118, 8286-8294.

(109) Trenins, G.; Willatt, M. J.; Althorpe, S. C. Path-Integral Dynamics of Water Using Curvilinear Centroids. J. Chem. Phys. 2019, 151, 054109. 
(110) Reddy, S. K.; Thiraux, R.; Rudd, B. A. W.; Lin, L.; Adel, T.; Joutsuka, T.; Geiger, F. M.; Allen, H. C.; Morita, A.; Paesani, F. Bulk Contributions Modulate the Sum-Frequency Generation Spectra of Water on Model Sea-Spray Aerosols. Chem 2018, 4, 1629-1644.

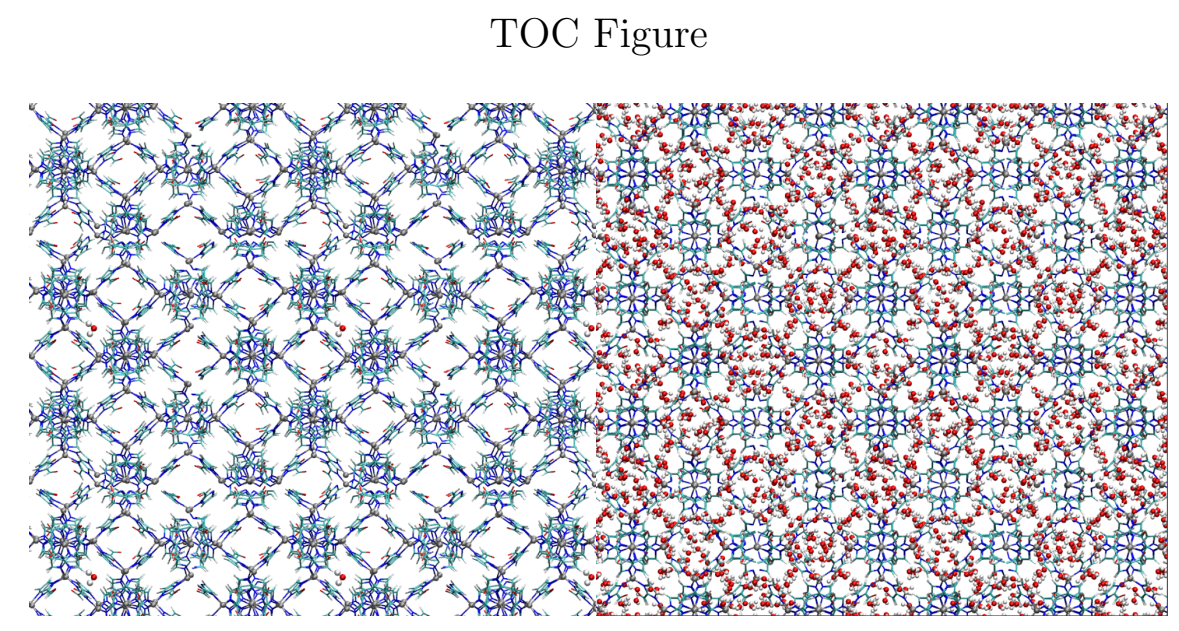

